\title{
Explicando la corrupción judicial en las cortes intermedias e inferiores de Chile, Perú y Ecuador*
} Santiago Basabe-Serrano**

\begin{abstract}
Resumen
Este artículo identifica las principales variables que explican la corrupción judicial en jueces inferiores y cortes intermedias de Chile, Perú y Ecuador. Refinando la metodología existente para la medición de la corrupción judicial e incorporando variables endógenas y exógenas al modelo, se propone que la formación profesional de los jueces, el respeto a la carrera judicial y el grado de fragmentación del poder en la arena política explican diferentes grados de corrupción judicial. A través de un modelo comparativo diacrónico y sincrónico de Chile, Perú y Ecuador, el artículo también constata que diseños institucionales en los que existen mayores instancias de impugnación o un mayor número de procedimientos, suelen ser más proclives a pagos ilegítimos u otras formas de corrupción judicial.
\end{abstract}

\begin{abstract}
This article identifies the main variables that explain judicial corruption in Chile, Peru, and Ecuador. Improving the current methodological strategies used to measure judicial corruption and incorporating endogenous and exogenous variables in the model, this article argues that legal training of the judges, respect for the judicial career, and the fragmentation of political power explain different degrees of judicial corruption. Through a comparative diachronic and synchronic research design of Chile, Peru and Ecuador, the article shows institutional designs with more legal steps will be more inclined to illegal payments or other types of judicial corruption.
\end{abstract}

Palabras clave: Corrupción judicial, justicia, Chile, Perú, Ecuador, calidad de jueces. Key words: Judicial corruption, justice, Chile, Perú, Ecuador, quality of judges.

* Esta investigación fue financiada por el Fondo de Desarrollo Académico de la Facultad Latinoamericana de Ciencias Sociales, Flacso Ecuador. Agradezco al equipo de investigación encargado de recolectar y procesar la información proveniente de las encuestas efectuadas en Santiago (Jorge Jocelín Almendras), Lima (Jorge Morel Salman) y Quito (Sergio García Rendón). Los comentarios de Tomás Mandl, John Polga-Hecimovich, María Isabel Rojas y dos revisores anónimos de la revista Perfiles Latinoamericanos contribuyeron a mejorar el presente artículo; no obstante, cualquier omisión es atribuible al autor.

** Doctor en Ciencia Política por la Universidad Nacional de San Martín, Buenos Aires, Argentina. Profesor-investigador titular del Departamento de Estudios Políticos de la Facultad Latinoamericana de Ciencias Sociales (Flacso) sede Ecuador. 
pesar de que la corrupción judicial ha sido uno de los temas de mayor interés para el campo de las políticas judiciales, no existe un consenso en cuanto a las variables que explican la presencia de dicho fenómeno. Tales desacuerdos se extienden también a la definición que mejor describe la corrupción judicial y a las estrategias metodológicas que permiten una medición empírica más fiable. Al final, el único criterio comúnmente aceptado es que los países tienen la imperiosa necesidad de combatir cualquier forma de corrupción, pues de eso depende la consolidación de las instituciones políticas (Selligson, 2002), el crecimiento económico, la disminución de la pobreza (Gupta et al., 2002) y el aumento de los niveles de inversión extranjera (Robertson y Watson, 2004). En otras palabras, se tiene mayor certidumbre respecto del comportamiento de la corrupción en general y de la corrupción judicial en particular, cuando se las estudia como variables independientes.

A fin de dar luces respecto a las variables que influyen de forma directa sobre los diferentes grados de corrupción judicial, este artículo propone un análisis comparado entre Chile, Perú y Ecuador. Para ello, propone utilizar como concepto de corrupción judicial la presencia de pagos ilegítimos a cambio de fallos judiciales. Aunque dicha definición podría omitir otro tipo de prácticas orientadas a alcanzar decisiones judiciales favorables a algún sujeto procesal, engloba buena parte de lo que constituye el fenómeno en estudio. Respecto al desarrollo del artículo, en la primera parte se discuten los principales hallazgos empíricos en relación con las variables que explican la corrupción judicial. En la segunda parte se ofrece un marco teórico a través del que se identifica el grado de distribución del poder político, la formación profesional de los jueces, el respeto a la carrera judicial y el número de instancias procedimentales como las variables clave para predecir la presencia de corrupción judicial. La tercera parte analiza las hipótesis que se desprenden del argumento teórico ya citado. En la cuarta parte se exponen las principales conclusiones de la investigación.

\section{Los estudios sobre corrupción judicial}

Dada la diversidad de enfoques asumidos para estudiar la corrupción judicial, para fines expositivos se los ha agregado en función del tipo de influencia que asumen como determinante de dicho fenómeno social. Así, se han considerado cinco conjuntos de variables: 1) de contexto social; 2) 
de funcionamiento interno de las cortes; 3) de naturaleza económica; 4) de origen institucional, y 5) de entorno político. A continuación, una reseña de los principales hallazgos empíricos de cada enfoque.

\section{Contexto social}

Una de las explicaciones más comunes respecto de la corrupción judicial se relaciona con la influencia que podría ejercer sobre la imparcialidad del juez el entorno en el que desempeña sus labores. Un primer enfoque señala que la tolerancia social a la corrupción es un factor clave para el incremento de las prácticas ilegítimas al interior de las cortes (Pepys, 2007). El argumento es que existen sociedades en las que ejecutar un acto de corrupción no es sancionado moral o éticamente sino que, por el contrario, se lo asume como parte de los códigos de comportamiento aceptados de cara a conseguir una justicia más ágil.

Otra de las variables citadas en esta dimensión refiere que la presencia de grupos criminales organizados influye en el aumento de la corrupción judicial (Buscaglia, 2007). Dada la cantidad de recursos económicos y de poder que poseen estas agrupaciones, no sólo las probabilidades de que los jueces acepten pagos a cambio de sentencias sino también que cambien sus opiniones ante amenazas contra su vida o la de su familia, irían en aumento. Esta explicación resulta plausible aunque sin la suficiente capacidad de generalización. En efecto, esta variable es esencial en sociedades en las que la existencia de grupos criminales organizados se encuentra plenamente identificada, pero deja de tener vigencia en aquellos países en los que existen altos niveles de corrupción judicial a pesar de la presencia residual de este tipo de agrupaciones. En el primer grupo estaría Colombia durante la década de los ochenta y noventa o algunos estados de México en la actualidad. ${ }^{1}$ En el segundo grupo se encuentran Ecuador, Paraguay o Venezuela.

Una tercera explicación tiene que ver con la presencia de mecanismos de transparencia y rendición de cuentas. El argumento es que la difusión de los fallos a través de páginas web oficiales contribuye a proporcionar incentivos negativos a la corrupción judicial (Rose-Ackerman, 2007). Uno de los casos en los que dicha conjetura ha sido verificada es Costa Rica, país en

Así lo reporta el informe 2011 de la Relatora Especial sobre la independencia de los magistrados y abogados del Alto Comisionado de las Naciones Unidas para los Derechos Humanos en su visita a México. El informe completo está disponible en: <http:/ / daccess-dds-ny.un.org/ doc/UNDOC/GEN/G11/129/36/PDF/G1112936.pdf?OpenElement>. 
el que a partir de la difusión de las sentencias de la Corte Suprema en su portal oficial, los niveles de corrupción judicial han descendido (Salazar y Ramos, 2007: 191). No obstante, el trabajo realizado por Voigt (2007: 299) concluye que la variable anotada presenta resultados insignificantes para explicar la corrupción judicial.

Del conjunto de variables de contexto social ya discutidas, se considera para la evaluación que este artículo propone la presencia de mecanismos de transparencia y rendición de cuentas. La existencia de hallazgos empíricos contradictorios - citados en el párrafo previo - respecto de la influencia de esta variable sobre la corrupción judicial torna interesante su retesteo pues contribuye de alguna manera a zanjar las discrepancias verificadas en la literatura especializada. Por otro lado, la tolerancia social a la corrupción y la presencia de grupos criminales organizados se dejan de lado por las razones que a continuación se explican.

En el caso de la tolerancia social a la corrupción, la ausencia de mediciones fiables dificulta derivar en conclusiones sólidas, que puedan ser asumidas con cierto grado de certidumbre. Además, la relación entre las variables tolerancia social a la corrupción y corrupción judicial presentarían un problema de circularidad - endogeneidad en términos cuantitativos difícil de resolver al menos en el plano cualitativo. En cuanto a la presencia de grupos criminales organizados como factor decisivo de la corrupción, las razones para su exclusión del modelo que este artículo ofrece tienen que ver con las limitaciones que la variable presenta para plantear conclusiones generalizables, y que han sido mencionadas en párrafos previos.

\section{Funcionamiento interno de las cortes de justicia}

Un segundo conjunto de variables explica la corrupción judicial a partir del análisis de la formación profesional de los jueces, la carga de trabajo que soportan y el respeto a la carrera judicial como mecanismo de ascensos y traslados dentro del Poder Judicial. En definitiva, la literatura considera que existen factores endógenos a las cortes que inciden sobre los diferentes grados de corrupción judicial. Respecto de la primera explicación, se dice que si los jueces tienen una buena formación académica, las probabilidades de que sucumban ante actos de corrupción van en descenso (Pepys, 2007: 6; Rose-Ackerman, 2007: 54; Hammergren, 2007: 145). El argumento es de orden cognitivo y apunta a que los actores que poseen un menor grado de información sobre un determinado tema son más propensos a orientar su conducta en función de las estrategias de disuasión o persuasión 
presentadas por quienes tienen un mayor conocimiento e información sobre el tema en discusión.

Por otro lado, entre los variados efectos que genera la sobrecarga de trabajo sobre los rendimientos del juez está el incremento de la corrupción judicial (Rose-Ackerman, 2007: 18; Salazar y Ramos, 2007: 192). El argumento que sostiene esta afirmación es que, dada la excesiva cantidad de juicios por resolver y la consiguiente incapacidad de dar atención a todas las causas, los jueces retrasan la toma de decisiones. Como consecuencia, los litigantes tienen incentivos suficientes para proponer un acto de corrupción, de cara a que su proceso sea resuelto prioritariamente. En definitiva, la cadena causal que propicia la corrupción judicial es la demora en la tramitación de los juicios. Nótese que en este caso el pago ilegítimo u otra forma de corrupción judicial busca obtener un despacho más ágil de las causas, lo que no implica necesariamente un direccionamiento del fallo judicial. Aunque lo dicho podría parecer un detalle, en términos analíticos genera implicaciones de diverso orden.

El respeto a la carrera judicial como mecanismo de ascensos y traslados constituye la última variable endógena a las cortes que explica la corrupción judicial (Hammergren, 2007). Dado que los jueces desean escalar posiciones al interior del Poder Judicial (Basabe-Serrano, 2011; Baum, 1997) y conocen que para ello es indispensable mantener una hoja de vida limpia, tales actores tendrán menos incentivos para incurrir en actos de corrupción. En sentido contrario, en aquellos países en los que los méritos alcanzados por los jueces en el desempeño de sus cargos no tienen el peso suficiente para obtener ascensos o traslados a tribunales mejor situados geográficamente, las probabilidades de que la corrupción judicial aumente son mayores (Pepys, 2007).

Respecto de las variables citadas, el modelo que este artículo propone considera a la calidad en la formación profesional de los jueces y al respeto a la carrera judicial como posibles explicaciones de los diferentes grados de corrupción judicial. Se deja de lado la variable relacionada con la sobrecarga de trabajo en virtud de que realizar un examen exhaustivo de su influencia sobre la corrupción judicial implica disponer de información precisa sobre los casos ingresados anualmente a cada una de las judicaturas y, desafortunadamente, en la mayor parte de países de América Latina es difícil encontrar datos con ese nivel de desagregación. En todo caso, al considerar dos de las tres variables halladas dentro del enfoque relacionado con el funcionamiento interno de las cortes de justicia se tiene una buena representación de los factores endógenos que podrían incidir sobre la corrupción judicial. 
Un tercer conjunto de variables apela a razones económicas como determinantes de la corrupción judicial. Así, se afirma que jueces con bajos salarios son más propensos a incurrir en actos de corrupción (RoseAckerman, 2007). No obstante, existe evidencia empírica en casos como el de Camboya en el que el aumento de salarios no generó variación alguna (Yang y Ehrichs, 2007) o el de Singapur, en el que aplicar dicha medida provocó efectos contrarios (Voigt, 2007). Por otro lado, se señala también que el PIB per cápita y el grado de apertura al mercado podrían generar incidencia sobre la corrupción en las cortes (Rose-Ackerman, 2007). Sin embargo, el trabajo de Voigt (2007: 298) ha constatado que la interacción de ambas variables explica apenas $46 \%$ de la variación en corrupción judicial mientras que la variable apertura al mercado por sí misma resulta insignificante.

Dado que existe suficiente evidencia empírica que relativiza la influencia que los factores económicos pueden generar sobre la corrupción judicial, el modelo ofrecido deja de lado el testeo de este tipo de variables. De hecho, junto al ya citado trabajo de Voigt (2007) en el que se señala la relación no significativa entre variables económicas y corrupción judicial, existe la investigación realizada por Sánchez Urribarrí (2008) en la que se llega a similares conclusiones luego del análisis de buena parte de los países de América Latina. En efecto, Sánchez Urribarrí constata que existe una relación negativa entre la variable salarios de los jueces de diferentes niveles y la percepción de corrupción. Más aún, el trabajo citado incluye los casos de Chile, Perú y Ecuador, países que constituyen las unidades de análisis del presente artículo.

\section{Factores institucionales}

El cuarto conjunto de variables utilizadas para explicar la corrupción judicial invoca razones de carácter institucional. Una de ellas asume que la concentración de competencias jurisdiccionales y administrativas en el mismo juez incentiva la presencia de actos de corrupción en las cortes (Buscaglia, 1999). Este argumento es el que, entre otros, dio paso a la creación de Consejos de la Judicatura en buena parte de los países de América Latina; no obstante, dos de los países con menores niveles de corrupción, como son Chile y Uruguay, carecen de este tipo de estructura institucional. ${ }^{2}$ En la misma línea,

2 Aunque en el caso chileno existe un órgano denominado Corporación Administrativa del Poder Judicial, integrado por cinco jueces supremos, y cuya finalidad es administrar el Poder Ju- 
aunque Ecuador y Bolivia poseen Consejos de la Judicatura, las mediciones realizadas por la organización Transparencia Internacional durante los últimos años sitúan a estos países con serios problemas de corrupción.

Hay que apuntar que el efecto que podría generar sobre la corrupción judicial la creación de Consejos de la Judicatura tiene que ver también con el alcance de las facultades otorgadas a este tipo de instituciones. En ese aspecto, un caso interesante de Consejo de la Judicatura con capacidades restringidas es el de El Salvador. ${ }^{3}$ En efecto, en ese país dicha estructura institucional se limita a seleccionar jueces inferiores mientras la Corte Suprema continúa concentrando las funciones administrativas del Poder Judicial. Quienes defienden la idea de que la creación de Consejos de la Judicatura influye sobre el descenso de la corrupción judicial parten del supuesto de que el diseño institucional marca claramente las diferencias entre las esferas de acción administrativa y jurisdiccional; $y$, a la par, otorga amplias capacidades a dichos órganos del Poder Judicial.

El segundo factor institucional tiene que ver con la incidencia del procedimiento que debe seguirse de cara a obtener una decisión judicial (Voigt, 2007; Rose-Ackerman, 2007; Pepys, 2007; Buscaglia, 1999). El argumento señala que a medida que aumenta el número de pasos a seguirse o que éstos no son lo suficientemente claros y objetivos, los costos de transacción de alcanzar un fallo judicial van en aumento (North, 1993). Luego, este tipo de costos adicionales - básicamente de tiempo - incentivan a que los usuarios del servicio judicial busquen una decisión oportuna a cambio de pagos ilegítimos o cualquier forma de corrupción judicial.

La creación de oficinas encargadas de perseguir los hechos de corrupción es otra de las variables institucionales identificadas por la literatura especializada. Aunque en Costa Rica esta medida ha dado buenos resultados (Salazar y Ramos, 2007: 191), existe evidencia empírica en la que se afirma que, probada en 63 países, dicha variable no tienen efectos significativos (Voigt, 2007: 299). Finalmente, hay autores que señalan que la presencia de diseños institucionales orientados a premiar a los jueces con una conducta ética constituye un incentivo para reducir la corrupción judicial (Pepys, 2007). Variables relacionadas con la conformación unipersonal o colegiada de los tribunales (Rose-Ackerman, 2007) o la prohibición de que

dicial, no se puede asimilar esta estructura institucional a lo que se conoce como Consejos de la Judicatura. Más allá de que sean solamente cinco de 22 jueces supremos chilenos los que conforman esta Corporación, el punto es que la esencia de los Consejos de la Judicatura es la diferenciación absoluta de las actividades administrativas y jurisdiccionales.

3 Agradezco por esta valiosa puntualización a uno de los revisores anónimos de la revista Perfiles Latinoamericanos. 
los jueces puedan desarrollar otro tipo de actividades han sido también identificadas dentro de los estudios realizados (Salazar y Ramos, 2007: 192). Lamentablemente, en ninguno de estos dos casos existe una clara identificación del vínculo causal entre tales variables y la corrupción judicial.

Del conjunto de variables de naturaleza institucional, este artículo considera para el análisis a las características del procedimiento que debe seguirse a fin de obtener una decisión judicial y a la presencia o no de incentivos institucionales para los jueces con una conducta ética. En ambos casos es relevante el modelo, pues se trata de variables que, a pesar de provenir de distintas vertientes del nuevo institucionalismo en ciencia política, permiten observar claramente cómo las reglas de juego pueden influir sobre el comportamiento de los jueces. En el primer caso, la referencia es a la perspectiva de la nueva economía institucional y los costos de transacción, mientras que, en el segundo caso, se hace alusión a los postulados básicos del institucionalismo analítico o de actores racionales.

Por otra parte, se deja de lado el análisis de las variables relacionadas con la presencia de Consejos de la Judicatura o de oficinas encargadas de perseguir actos de corrupción pues, como se ha manifestado, existe evidencia empírica suficiente que da cuenta de la ausencia de correlación entre la presencia de este tipo de diseños institucionales y la disminución del fenómeno que interesa estudiar. Finalmente, la estructura unipersonal o colegiada de los tribunales y la prohibición de que los jueces puedan ejercer actividades distintas al ejercicio de la judicatura también son apartadas del modelo puesto que, como también se ha señalado, no existe un argumento teórico lo suficientemente convincente que permita vincular a estas variables con el aumento o disminución de la corrupción judicial.

\section{Entorno político}

El último conjunto de variables tiene relación con el grado de dispersión del poder en la arena política. El argumento que aquí se plantea es que, a medida que el poder político se encuentra compartido entre un mayor número de actores, existirían mayores probabilidades de un "buen gobierno" al interior del ejecutivo, la legislatura y las cortes (Montinola y Jackman, 2002). La idea central en este caso es que si el poder político se encuentra más fragmentado, las probabilidades de que existan diferentes intereses en los poderes del Estado es mayor, facilitando así el mutuo control y chequeo de sus acciones y desincentivando la presencia de conductas corruptas. Aunque Melgar y Peña (2007: 214) han verificado para el caso de Guatemala la validez de esta 
hipótesis, el trabajo de Voigt (2007) arroja resultados poco significativos en su estudio realizado para 63 países. ${ }^{4}$

Dadas las tensiones en cuanto a hallazgos empíricos, este artículo incluye la variable ya discutida dentro del modelo propuesto. Además, se justifica considerar en el testeo la influencia de la fragmentación del poder político sobre la corrupción judicial pues, en general, la relación entre política y justicia se ha tratado mayoritariamente desde la perspectiva de la independencia judicial y la capacidad de los políticos de influir sobre las decisiones de los jueces. En ese aspecto, observar cómo las modificaciones en la correlación de fuerzas en el entorno político inciden sobre el comportamiento de los jueces, no sólo en la toma de decisiones judiciales controvertidas sino también en aquellas que afectan a la ciudadanía en general, conlleva a una comprensión más amplia de los desempeños del Poder Judicial.

\section{Una propuesta teórica para evaluar la corrupción judicial}

Como se ha observado, existe una serie de hipótesis que explicarían la emergencia e incluso sedimentación de la corrupción judicial. Sin embargo, los ejercicios de verificación empírica son residuales y, cuando existen, resultan poco sistemáticos. En dicho escenario, este artículo se propone probar de forma unificada los principales aportes hallados en los trabajos realizados hasta el momento. Para ello, se utiliza una metodología comparada en la que destaca una medición de la corrupción judicial que, en relación con las realizadas hasta ahora, ofrece resultados más sólidos y robustos. Como ya se ha anotado, para establecer las variables explicativas que serán analizadas se tomó en cuenta a aquellas que han sido verificadas al menos en algún caso y que, por otro lado, presentan razonables vínculos de causalidad respecto a la corrupción judicial.

En primer lugar, se considera el efecto que puede generar sobre la corrupción judicial los mecanismos de difusión de las decisiones judiciales a través de páginas web oficiales. En ese aspecto, si los poderes judiciales - fundamentalmente las cortes supremas - publican sus sentencias y demás decisiones importantes existiría una menor propensión a actos de corrupción judicial. En términos de hipótesis se propone que a mayor di-

4 No obstante, se debe considerar que el trabajo de Voigt asume como fragmentación del poder político el número de jugadores de veto existente en la arena política, en la línea del argumento de veto players propuesto por George Tsebelis (2002). Lo dicho genera sesgo en la medición efectuada. 
fusión de las sentencias judiciales, las probabilidades de corrupción judicial irían en descenso.

Por otro lado, la calidad en la formación profesional de los jueces constituiría un elemento influyente en la propensión a actos de corrupción judicial. Así, en aquellos países en los que los jueces gozan de un mejor nivel académico los incentivos para incurrir en hechos de corrupción judicial serían menores. Lo dicho se plantea, fundamentalmente, por el impacto que tales actos generan sobre el prestigio y estatus de los juzgadores. En el plano conjetural se diría que a medida que los jueces tienen una mayor capacitación profesional y académica para el ejercicio de sus funciones, las probabilidades de actos de corrupción judicial van en descenso.

En tercer lugar, el respeto a la carrera judicial como mecanismo de ascensos y traslados constituye otra de las variables que incidirían sobre la corrupción en las cortes. En otras palabras, se conjetura que los países en los que los méritos y deméritos de los jueces son valorados de forma contundente para tomar decisiones administrativas dentro del Poder Judicial, son los países en los que las cortes de justicia son menos proclives a actos de corrupción judicial. A manera de hipótesis se puede decir que a medida que la carrera judicial es más valorada como mecanismo de provisión de ascensos y traslados para los jueces, las probabilidades de corrupción judicial van en descenso.

La cuarta variable tiene que ver con las características del procedimiento utilizado para el trámite de los procesos legales. Específicamente, la complejidad y exceso de pasos constituirían un incentivo negativo al comportamiento ético de los jueces. En otras palabras, la ausencia de objetividad y lo engorroso de los procedimientos utilizados darían paso a que los usuarios del servicio judicial se vean motivados a ofrecer pagos ilegítimos o cualquier acto de corrupción de cara a obtener decisiones judiciales. En formato de hipótesis se diría que a medida que aumenta el número de trámites o procedimientos necesarios en una causa legal, las probabilidades de corrupción judicial van en aumento.

Por otro lado, la presencia o no de incentivos institucionales a los jueces que no incurren en actos de corrupción es también parte de la valoración. $\mathrm{Al}$ respecto se dirá que la existencia de disposiciones legales o constitucionales que premien a los jueces con un comportamiento ético disminuye las probabilidades de corrupción judicial. Finalmente, una variable adicional sometida a estudio tiene que ver con el grado de distribución del poder político. Se puede conjeturar que a medida que el poder político se encuentra más fragmentado, las probabilidades de corrupción judicial van en descenso. 
Para probar las hipótesis descritas se analiza el comportamiento de los jueces inferiores y de cortes intermedias de Chile, Perú y Ecuador. Estos países resultan interesantes para la comparación, pues en las mediciones realizadas por Transparencia Internacional y otras organizaciones afines, sus niveles de corrupción - en términos generales - suelen ser muy diferentes, a pesar de que comparten una tradición jurídica similar, como es el sistema de origen romano-germánico o continental. En el plano metodológico, esta selección de unidades de análisis garantiza varianza y evita sesgos de variable trunca al dejar de lado unidades que contienen solamente valores extremos en la variable dependiente.

En relación con el corte temporal, se tomaron en cuenta tres episodios históricos clave para la vida judicial de cada país. Dicha periodización se realizó en función de los cambios tanto en la correlación de fuerzas políticas como en la distribución del poder en cada uno de los países. ${ }^{5}$ En el caso chileno, el primer corte abarca del 11 de marzo de 1990 al 11 de marzo de 1994. Éste es el periodo del retorno al régimen democrático y en el que aún se podría notar la influencia de enclaves autoritarios (Garretón, 2002) sobre la corrupción judicial. El segundo momento va del 11 de marzo de 1994 al 31 de diciembre de 1997 y corresponde a la época de asentamiento de la democracia que debería ir de la mano de mayor transparencia en las cortes. Por último, el tercer periodo va desde el $1^{\circ}$ de enero de 1998 hasta junio de 2011. La puesta en vigencia de un proceso de reforma judicial, iniciado en el área penal, daría pautas respecto de la verificación de la variable relacionada con la disminución de los trámites procesales y su relación con la corrupción judicial.

Para el caso peruano, el primer periodo va del 28 de julio de 1985 al 5 de abril de 1992 y corresponde a la época de supremacía política del Partido Alianza Popular Revolucionaria Americana (APRA) por lo que la ausencia relativa de distribución del poder político en esta época podría haber generado efectos sobre la corrupción judicial. El segundo corte va del 5 de abril de 1992 al 22 de noviembre de 2000. Esta etapa inicia con el "autogolpe" del presidente Fujimori y concluye con su renuncia. Aquí las reglas elementa-

5 La periodización en cada uno de los países con base en la presencia de cambios en la distribución del poder político permite comparar los casos sin necesidad de recurrir a cortes temporales regulares anuales que, desde el punto de vista del funcionamiento judicial, resultan poco útiles. Agradezco por este comentario y sugerencia a uno de los revisores anónimos de la revista Perfiles Latinoamericanos. 
les del régimen democrático se pusieron en duda y, como consecuencia, la corrupción judicial debería presentar un incremento. Finalmente, el último periodo va del 22 de noviembre de 2000 hasta junio de 2011 y coincide con el retorno a la democracia y la puesta en vigencia de una efectiva reforma judicial. Por la diversidad de esferas tocadas por ese proceso de cambio en el Poder Judicial, se esperaría que la corrupción judicial haya disminuido durante estos años.

En el caso del Ecuador, la primera etapa va de octubre de 1997 a diciembre de 2004. Este momento histórico corresponde a la designación de jueces de la Corte Suprema por periodos vitalicios, lo que habría otorgado estabilidad al Poder Judicial, un incremento en la calidad de los jueces de las diferentes instancias y una modificación del poder político no sólo en la arena judicial sino también política. Como consecuencia, se presumiría que la corrupción judicial tendería a no ser tan alarmante. El segundo periodo va de diciembre de 2005 a diciembre de 2008. Esta etapa sigue a un periodo de más de un año de inercia institucional durante el que Ecuador careció tanto de Corte Suprema como de Tribunal Constitucional (BasabeSerrano y Polga Hecimovich, 2013; Basabe-Serrano, 2012). En lo de fondo, no se deberían esperar mayores variaciones en cuanto a los niveles de corrupción judicial observados en el periodo previo.

El último corte temporal analiza el periodo que va entre diciembre de 2008 y junio de 2011. Ese momento es correlativo con la vigencia de una nueva Constitución Política y fundamentalmente con el aparecimiento de un proceso político con rasgos hegemónicos (Basabe-Serrano et al., 2010). En tal escenario, en el que el personalismo y la polarización han diezmado la distribución del poder político (Pachano, 2010; De la Torre, 2010; Conaghan, 2008), se podría conjeturar que la corrupción judicial ha ganado terreno. De hecho, recientes trabajos sobre el caso ecuatoriano presentan evidencia empírica que da cuenta de la mayor ductilidad del Poder Judicial frente a la orientación que se desea dar desde el Poder Ejecutivo a determinados casos judiciales en los que de por medio existen intereses políticos en juego (Conaghan, 2012).

Independientemente de que los cortes temporales identificados para cada país no coincidan exactamente en cuanto a los años analizados, la comparación no se ve afectada, pues cada periodo corresponde a diferentes momentos institucionales de los respectivos poderes judiciales. ${ }^{6}$ Como se ha señalado en la propuesta teórica de este artículo, la corrupción judi-

6 La ausencia de uniformidad en los cortes temporales analizados se conoce en la metodología cuantitativa como panel desbalanceado (unbalanced panel). 
cial guarda relación con factores endógenos a las cortes pero también con otros de naturaleza política, social e institucional Por ello, variaciones en el sistema político sirven no sólo para identificar metodológicamente los distintos periodos de la vida institucional de las cortes sino también para valorar tanto las variables endógenas al Poder Judicial como aquéllas provenientes del entorno.

\section{El estado de la corrupción judicial en Chile, Perú y Ecuador}

Uno de los principales desafíos de la presente investigación constituye la definición de la estrategia para medir la corrupción judicial. Al respecto, la medición más común es la que efectúa el World Economic Forum y que se difunde año con año a través del Global Competitiveness Report. Allí se consulta a personas de negocios de los diferentes países sobre la frecuencia con la que ocurren pagos irregulares en las decisiones judiciales. Aun cuando este artículo también recurre a encuestas, los informantes son expertos en temas judiciales: abogados, ex jueces y docentes universitarios. A diferencia de los encuestados por el World Economic Forum, los actores a quienes esta investigación consultó son personas que por sus actividades tienen una visión más amplia de la corrupción judicial y no limitada a sus propias vivencias, como sería el caso de los empresarios.

Sin embargo, es necesario puntualizar que a pesar de que los informantes que este artículo consultó conocen de mejor forma el sistema judicial que los empresarios, también responden en función de intereses particulares. Lamentablemente, ése es un sesgo que es necesario asumir, pues no deja de estar presente en cualquier técnica de encuestas. Además, un contraargumento a la medición planteada es que los informantes podrían ofrecer respuestas sesgadas en el sentido de favorecer a las instituciones judiciales por su cercanía y vinculación con el contexto jurídico de cada país. No obstante, comparando las mediciones más recientes que aquí se entregan con las del índice del Global Competitiveness 2011-2012 - pues no existe dicho indicador para las primeras periodizaciones planteadas en este artículo - se observa que los hallazgos empíricos de esta investigación, por el contrario, son menos favorables a los poderes judiciales de los tres países que los ofrecidos por el índice ya indicado.

Así, el índice del Global Competitiveness 2011-2012 en su medición de corrupción judicial coloca a Chile con un valor de 8.42/10 mientras que los resultados de la encuesta aquí ofrecida marcan una puntuación de 7.60/10. En el caso de Perú, el índice citado coloca a ese país con 6.14/10 cuando el 
resultado de las encuestas elaboradas para este artículo lo ubica en 5.00/10. Finalmente, para Ecuador el Global Competitiveness 2011-2012 otorga una puntuación de 4.85/10 mientras en esta medición alcanza 3.76/10. Como se observa, y en tensión con el contraargumento que se podría asumir, los expertos en temas judiciales consideran que la corrupción judicial en Chile, Perú y Ecuador es mayor que la que perciben las personas de negocios. ${ }^{7}$ Como se dijo, no es posible efectuar la comparación para el resto de periodizaciones que este artículo plantea y por tanto tampoco es viable obtener valores que resulten de la media de ambas mediciones.

Una ventaja adicional de la medición que aquí se ofrece radica en que la muestra captura ciento cincuenta encuestas $(n=150)$ recolectadas en cada una de las capitales de los países analizados - 50 en Santiago, 50 en Lima y 50 en Quito - . De esa forma, el número de observaciones es más significativo que el utilizado en mediciones previas, que suelen recurrir a pocos encuestados por país. ${ }^{8}$ Desde luego, aunque el número de encuestas utilizadas en este trabajo mejora la representatividad respecto a trabajos similares, la muestra no debe ser considerada como estadísticamente representativa. Capturar un número de encuestas que cumpla con esta condición implica contar previamente con un universo definido de abogados, ex jueces y docentes universitarios con conocimientos sobre los desempeños institucionales del Poder Judicial, lo que en América Latina no es posible identificar. ${ }^{9}$

Ante esta falencia, se recurrió a la técnica de muestreo por saturación. Dada la diferencia poblacional observada en Santiago, Lima y Quito, que de alguna manera debería estar correlacionada con el número de expertos judiciales que se podría encuestar, el muestreo por saturación generó los mejores efectos en la ciudad de Quito. ${ }^{10}$ La batería de preguntas se tomó de forma personal entre los meses de abril y junio de 2011. A fin de ase-

7 La medición del Global Competitiveness 2011-2012 va en una escala de 7 a 1 (donde 7 es un país ausente de corrupción judicial). Para fines de la comparación propuesta se estandarizaron estos valores a una escala de 10 a 1 que utiliza esta investigación.

El aporte de mediciones como las del Doing Business consiste en que, a pesar de considerar pocas encuestas por país, abarcan buena parte de los estados a nivel mundial con lo que la posibilidad de realizar comparaciones de mayor amplitud se viabiliza.

9 Una opción para capturar un universo definido de expertos judiciales es el registro generado en los denominados Colegios de Abogados. No obstante, el problema que aquí se presenta es que existen muchos profesionales del Derecho que a pesar de hallarse inscritos como tal, no ejercen su profesión o lo hacen en áreas de especialización - como la propiedad intelectual o el derecho societario - en las que no hay una vinculación sistemática con el Poder Judicial.

Acorde con datos de las oficinas de censos de cada país, Santiago de Chile tiene una población de 6,683,680 habitantes (medición del año 2012), Lima tiene 7,605,742 habitantes (medición de 2008), y Quito registra 2,239,199 habitantes (medición de 2010). 
gurar la fidelidad de los datos obtenidos se garantizó el anonimato a los informantes. Finalmente, la selección de encuestados procuró mantener equilibrio de género y a la par una diversidad de tendencias ideológicas.

Sobre este último punto, la encuesta incluyó una pregunta relacionada con autoubicación ideológica de los informantes. Con dichos valores se extrajo la media de cada país, conforme consta en la columna derecha de la tabla No 1. Como se observa, la media ideológica de los encuestados en Chile es de 5.22/10 mientras que en Perú arriba a 4.95/10; y, en Ecuador, a 4.92/10. Tales resultados reflejan la existencia de posiciones moderadas en los informantes y el consiguiente efecto sobre la disminución de sesgos atribuibles a tales personas. Sin embargo, la posibilidad de que las respuestas se hayan racionalizado en función del recuerdo de lo ocurrido en periodos previos se mantiene. Dada la inexistencia de estrategias alternativas para medir la corrupción judicial a lo largo del tiempo, es necesario asumir este error de medición como parte de la incertidumbre propia de la investigación científica.

La pregunta planteada en los tres países fue similar y se refirió fundamentalmente a la presencia de pagos ilegítimos a cambio de fallos judiciales en las cortes inferiores e intermedias. Por tanto, éste es el concepto de corrupción judicial que se asume como eje articulador del análisis empírico presentado. Nótese que la percepción de corrupción judicial que se obtuvo concentra su atención en jueces distintos a los de las cortes supremas. Esta focalización es valiosa pues, a diferencia de investigaciones en las que se pregunta respecto a percepciones de corrupción judicial en general, la especificidad que aquí se ofrece da cuenta de la realidad de aquellas cortes que, por la naturaleza de casos que tramita, están más cerca de la cotidianidad de la ciudadanía. Con los resultados obtenidos se obtuvo una media para los diferentes cortes temporales en cada uno de los países analizados. La Tabla No. 1 describe los hallazgos empíricos ya reportados.

En el caso chileno, los índices de corrupción judicial han mejorado con el paso del tiempo. Aunque en el primer periodo (1990-1994) el indicador muestra falencias (5.44/10), durante el segundo corte temporal (1994-1997) se observa ya una mejoría, aunque no del todo ostensible (6.20/10). Es en el tercer periodo (1998-2011) en el que se constata un despunte del indicador de corrupción judicial (7.60). Lo dicho da cuenta de la presencia de factores que han ejercido una influencia importante en los últimos años sobre el comportamiento ético de los jueces chilenos. No obstante, hay que anotar que en términos generales las calificaciones recibidas por Chile en temas de corrupción han sido siempre aceptables si se las compara con las alcanzadas por el resto de países de América Latina. 
Tabla No. 1

Percepción de corrupción judicial en Chile, Perú y Ecuador

\begin{tabular}{lcccc}
\hline País & Periodo No. 1 & Periodo No. 2 & Periodo No. 3 & $\begin{array}{c}\text { Ubicación ideológica de } \\
\text { encuestados }\end{array}$ \\
\hline Chile & 5.44 & 6.20 & 7.60 & 5.22 \\
Perú & 3.39 & 2.20 & 5.00 & 4.95 \\
Ecuador & 5.78 & 5.52 & 3.76 & 4.92 \\
\hline
\end{tabular}

Fuente: Encuestas realizadas a expertos.

El rango va de " 10 " que significa ausencia total de corrupción judicial hasta " 1 ", que significa presencia total de corrupción judicial.

En el caso peruano la trayectoria de la corrupción judicial es diversa a lo ocurrido en Chile. Durante el primer periodo (1985-1992) el indicador revela una fuerte injerencia de pagos ilegítimos a cambio de fallos judiciales (3.39/10). No obstante, el periodo siguiente (1992-2000) es de rendimientos aún más deficitarios (2.20/10) lo que refleja el estado en el que se encontraba el Poder Judicial durante el gobierno del presidente Alberto Fujimori. Finalmente, en el último momento histórico analizado (2000-2011) hay un repunte importante del indicador (5.00/10). Aunque sin llegar a niveles como el chileno, la palpable recuperación de la transparencia al interior de las cortes peruanas constituye un buen presagio para el futuro del Poder Judicial de ese país.

El Ecuador es el país que presenta resultados más preocupantes, pues la tendencia es decreciente a lo largo de todo el periodo analizado. Durante el primer corte temporal (1997-2004) los niveles de corrupción son medios $(5.78 / 10)$ y guardan relación con los observados en Chile luego del retorno a la democracia o en Perú actualmente. En el segundo periodo (2005-2008) se verifica un incremento de la corrupción judicial aunque sin mayor variación respecto a los valores observados previamente (5.52/10). Lamentablemente, en el último corte temporal (2008-2011) es donde se observa un deterioro severo en la conducta ética de los jueces ecuatorianos (3.76/10). Aunque en sentido contrario al observado en el caso chileno, este dato sirve para indagar en torno a los factores que han ejercido influencia sobre esta importante variación en la corrupción judicial de ese país.

\section{Explicando la corrupción judicial en Chile, Perú y Ecuador}

Con la descripción previa, a continuación se valora la correlación existente entre corrupción judicial y aquellas variables que en este artículo se proponen como explicativas. Para ello, se plantea un modelo de investigación en el que 
se infiere causalidad a partir de la identificación de los factores que asumen valores diferentes en cada unidad de análisis y en cada periodo temporal (Przeworski y Teune, 1970). Para medir las seis variables explicativas de la corrupción judicial se usaron diversas estrategias metodológicas.

Para triangular la información, adicionalmente se realizaron entrevistas semiestructuradas con expertos en las ciudades de Santiago, Lima y Quito. Del total de entrevistados, la gran mayoría no habían sido considerados para las encuestas ya referidas. Se realizaron 87 entrevistas (26 en Santiago, 29 en Lima y 32 en Quito), todas tomadas de forma personal entre los meses de abril y julio de 2011. Puesto que muchos de los entrevistados litigan frecuentemente en las cortes, se les garantizó reserva de sus identidades. Al igual que en las encuestas, se buscó mantener la equidad de género y posicionamientos ideológicos variados. Esto último se consiguió seleccionando expertos cuya práctica profesional se focaliza en diversas áreas del Derecho - laboral con enfoque sindicalista, societario, derechos humanos, propiedad intelectual.

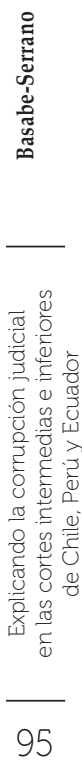

Difusión de los fallos judiciales a través de sitios web oficiales

Como se señaló, la difusión a través de internet de las sentencias y, en general, de las decisiones asumidas por los jueces constituye un mecanismo de transparencia que aporta a la reducción de la corrupción judicial. Con ello, para observar empíricamente dicha variable se recurrió al análisis de las páginas web oficiales del Poder Judicial de los tres países. La información obtenida posteriormente fue triangulada con la proveniente de las entrevistas semiestructuradas. Por las características de esta variable - internet tiene plena difusión en América Latina recién a mediados de la década de los noventa - solamente se ha conseguido información fiable para el último periodo de análisis de cada país. Por tanto, la comparación que se ofrece es de naturaleza sincrónica.

Así, de los casos sometidos a análisis solamente Chile posee un sistema de información completo e integrado de publicación de los fallos judiciales. ${ }^{11}$ En efecto, la página oficial del Poder Judicial de ese país permite conocer el estado actual de los juicios iniciados en cualquiera de las siete regiones chilenas. Además cuenta con un buscador por fechas, número de expedientes y nombres de las partes procesales, lo que da mayor facilidad al usuario para conocer la marcha de los casos. En Perú y Ecuador la

11 A través de la página web del Poder Judicial: <www.poderjudicial.cl>. 
publicación de fallos es parcial en lo que se refiere a las cortes de máxima instancia mientras que, respecto a juzgados inferiores, es muy poco lo que se puede obtener a través de internet. ${ }^{12}$

\section{Calidad de los jueces}

Para medir la calidad de los jueces en Chile, Perú y Ecuador se incluyó en la encuesta una pregunta al respecto, de donde se obtuvo la media de cada periodo histórico para cada país. ${ }^{13}$ Los resultados de dicha medición constan en la Tabla No 2. Como se puede observar, tanto en el caso chileno (5.33/10; $5.75 / 10 ; 6.62 / 10)$ como en el peruano $(3.21 / 10 ; 2.89 / 10 ; 4.79 / 10)$ existe una mejoría en cuanto a la calidad en la formación de sus jueces. Desde luego, en Chile este proceso es de permanente ascenso mientras que en Perú se verifica luego del retorno a la democracia en el año 2000. En una dirección opuesta se encuentra Ecuador $(5.98 / 10 ; 5.28 / 10 ; 3.98 / 10)$, país en el que a partir de 2008 - último periodo analizado - los niveles de calidad de sus jueces ha sufrido un grave menoscabo.

Tabla No. 2

Calidad en la formación de los jueces en Chile, Perú y Ecuador

\begin{tabular}{cccc}
\hline País & Periodo No. 1 & Periodo No. 2 & Periodo No. 3 \\
\hline Chile & 5.3 & 5.75 & 6.62 \\
Perú & 3.21 & 2.89 & 4.79 \\
Ecuador & 5.98 & 5.28 & 3.98 \\
\hline
\end{tabular}

Fuente: Encuestas realizadas a expertos.

El rango va de " 1 " que corresponde a una deficiente calidad de los jueces y va hasta "10", que corresponde a una excelente calidad de los jueces.

En un mundo ideal, se podrían vincular los resultados de la medición planteada con otros datos que abonen a un referente empírico más preciso respecto al concepto calidad de los jueces. Una opción válida es la observación de los títulos de posgrado de los jueces. Aunque dicha información se la podría obtener en el caso chileno, el acceso a estos datos

12 En el caso ecuatoriano existen algunas páginas web de cortes de segunda instancia que publican los avances de las causas judiciales; no obstante, son absolutamente residuales las provincias que proveen ese servicio.

13 El protocolo íntegro de la encuesta puede ser requerido a través de la página web: <www.santiagobasabe.com>. 
se dificulta en el caso peruano, mientras que en Ecuador es una tarea casi imposible de lograr debido al hermetismo que existe al respecto en el Consejo de la Judicatura de ese país. ${ }^{14}$ Por otro lado, una crítica que se podría plantear a la medición que este artículo propone es que los datos están afectados por un problema de endogeneidad, en la medida en que es probable que los encuestados que califican a sus cortes con un alto nivel de corrupción al mismo tiempo otorguen una puntuación baja a la calidad de los jueces o viceversa.

Sobre este último tema, al comparar los resultados obtenidos para el caso chileno en las mediciones tanto de corrupción judicial como de calidad de los jueces, no se observan los posibles efectos de la endogeneidad mencionada. En el caso peruano, aunque en las dos primeras periodizaciones existe una relación inversa entre las dos mediciones - lo que en principio serviría solamente como un indicio de endogeneidad - , en los valores del periodo final tanto las calificaciones de la corrupción judicial como las de calidad de los jueces se mantienen en un nivel medio. En el caso de Ecuador, solamente en el periodo final se observan valores que indican altos niveles de corrupción judicial acompañados de baja calidad en la formación de los jueces. Las mediciones de los dos periodos previos no dejan entrever, al menos intuitivamente, la existencia de problemas de endogeneidad.

\section{Respeto a la carrera judicial como mecanismo de promoción}

Para medir el grado de respeto a la carrera judicial existente en los tres países se incluyó en la misma encuesta una pregunta al respecto, obteniéndose posteriormente las medias de cada medición para cada país. Como se observa en la Tabla No. 3, en Chile hay una tendencia recurrente hacia una mayor institucionalización de la vida burocrática del Poder Judicial, lo que se refleja en la atención brindada a la carrera judicial dentro de los criterios básicos para la obtención de promociones y ascensos $(5.63 / 10 ; 6.26 / 10 ; 7.22 / 10)$. En la misma dirección se sitúan los resultados obtenidos para el caso peruano $(3.14 / 10 ; 2.28 / 10 ; 5.48 / 10)$, fundamentalmente durante el último periodo analizado. Finalmente,

14 De hecho, al solicitar información relacionada con las hojas de vida de los jueces ecuatorianos, el Consejo de la Judicatura se ha negado categóricamente a atender dicho pedido aduciendo que se trata de información personalísima de los jueces y que, por tanto, es un caso en el que el libre acceso a la información no procede. 
los datos reportan un retroceso importante en este campo para el caso ecuatoriano (5.58/10; 5.18/10; 3.78/10). De hecho, a partir de 2008 los resultados obtenidos dan cuenta de un proceso de desinstitucionalización de la carrera judicial, observable en el poco interés dado a este campo al momento de tomar decisiones administrativas relacionadas con cambios o ascensos de los jueces.

Tabla No. 3

Respeto a la carrera judicial en Chile, Perú y Ecuador

\begin{tabular}{cccc}
\hline País & Periodo No. 1 & Periodo No. 2 & Periodo No. 3 \\
\hline Chile & 5.63 & 6.26 & 7.22 \\
Perú & 3.14 & 2.28 & 5.48 \\
Ecuador & 5.58 & 5.18 & 3.78
\end{tabular}

El rango va de " 1 " que corresponde a una ausencia absoluta de importancia dada a la carrera judicial al momento de promover jueces inferiores hacia juzgados superiores y va hasta 10 , que corresponde a una importancia prioritaria y definitiva dada a la carrera judicial al momento de promover jueces inferiores hacia juzgados superiores. Fuente: Encuestas realizadas a expertos

Los datos mencionados fueron triangulados posteriormente con información proveniente de las entrevistas semiestructuradas que se realizaron a expertos no considerados para la realización de la encuesta. Aunque los datos relacionados con esta variable podrían ser más consistentes si se toman en cuenta referentes empíricos como el número de jueces promovidos a través de concursos públicos o la cantidad de jueces destituidos luego de un sumario administrativo, el problema del acceso a ese tipo de información vuelve a ser una barrera difícil de superar. Como se dijo al discutir la medición de la calidad de los jueces, en un mundo ideal este tipo de datos deberían estar al alcance de la ciudadanía. En la mayoría de países de América Latina, salvo el caso chileno, uruguayo o costarricense, las oficinas de control administrativo de las cortes supremas o los Consejos de la Judicatura manejan la información relacionada con el desempeño de los jueces bajo un secretismo infranqueable.

Características del procedimiento judicial

Para capturar cuán engorroso y complejo es el trámite establecido para la sustanciación de los juicios, se analizaron dos tipos de procedimientos, uno en materia penal y otro en materia civil. En el primer caso se consideró el juicio penal ordinario, aplicable a la mayoría de delitos, mientras que en 
el segundo caso se tomó en cuenta al juicio ejecutivo, cuya finalidad es conseguir el cumplimiento de obligaciones previamente aceptadas. Dadas las connotaciones sociales y políticas de estos dos campos del Derecho, se los asumió como un buen proxy de la variable referida. Para la medición se recurrió a los códigos de Procedimiento Penal y de Procedimiento Civil de cada país, analizando la cantidad de recursos que se pueden interponer a las decisiones judiciales definitivas y las etapas del proceso hasta llegar a un fallo definitivo, respectivamente. Los plazos establecidos para la resolución de los recursos o para transitar de un estado procesal a otro no son parte de la valoración pues, más allá de la declaración normativa, suelen decir muy poco de los hechos fácticos. Para triangular los datos se verificó su validez a través de las entrevistas realizadas con los expertos nacionales.

Respecto de los recursos que se pueden interponer dentro del proceso penal, en el caso chileno, la sentencia dictada por un tribunal de juicio oral es susceptible solamente del recurso de nulidad. ${ }^{15}$ De su lado, en la legislación del Perú los fallos definitivos pueden ser impugnados vía casación y apelación. Finalmente, en Ecuador la sentencia puede ser recurrida a través de las dos formas previamente indicadas más el recurso de nulidad. Además, desde la Constitución de 2008, es factible interponer el recurso extraordinario de protección, que se sustancia ante la Corte Constitucional. Dicho recurso procede solamente contra sentencias o autos definitivos en los que se observen violaciones, por acción u omisión, de derechos reconocidos en la Constitución. ${ }^{16}$

Cabe anotar que la simplificación del procedimiento en Chile guarda relación con la reforma judicial de 1998, plasmada en su nuevo Código de Procedimiento Penal del año 2000 (Carbonell, 2007, 2008; Harasic, 2007: 189). En el caso peruano las reformas datan de 2004, mientras que en el Ecuador la incorporación del recurso de apelación como vía impugnatoria a la sentencia se verificó en el año 2009. Antes de ese año en dicho país solamente se permitían los recursos de nulidad y casación. Por tanto, aunque la Tabla No. 4 (pág. 76)presenta una comparación sincrónica de los tres países, da cuenta de los cambios reales suscitados durante los tres periodos que este artículo analiza. En general, mientras Chile se encuentra en un proceso de agilitar su proceso penal en Ecuador sucede lo contrario.

En cuanto al juicio ejecutivo chileno, luego de la calificación de la demanda, con la orden judicial de pago - auto de pago - y la contestación

15 En la legislación chilena, el recurso de nulidad en materia penal se parece a lo que constituye la Casación en Perú y Ecuador.

16 En conformidad con lo que dispone el art. 94 de la Constitución del Ecuador. 
de parte del deudor, el acreedor tiene cuatro días para dar una respuesta. Con los contra argumentos del acreedor o aun a falta de ellos, el juez dicta sentencia tanto si considera inadmisibles los argumentos del deudor - excepciones - como si a su criterio no es necesario conceder un plazo para la práctica de pruebas. En caso de requerir pruebas, el juez concede 10 días para ello, luego de lo que dicta sentencia. ${ }^{17}$ En el caso peruano, el juez califica la demanda, dicta la orden judicial de pago y, con la contestación del deudor, concede al acreedor tres días para escuchar sus argumentos. A diferencia del caso chileno, en el que el juez puede dictar sentencia directamente y es potestativo el otorgamiento del plazo probatorio, en Perú es imprescindible la celebración de una audiencia en la que se reciben las pruebas de ambas partes. En esta misma diligencia judicial el juez dicta sentencia o, en su defecto, luego un plazo perentorio de diez días.

Tabla No. 4

100 Recursos procesales a la sentencia penal en Chile, Perú y Ecuador

\begin{tabular}{cccc}
\hline & & Países & \\
\hline Recursos & Chile & Perú & Ecuador \\
\hline Casación & $\mathrm{X}$ & $\mathrm{x}$ & $\mathrm{X}$ \\
Apelación & $\mathrm{X}$ & $\mathrm{X}$ \\
Nulidad & & $\mathrm{X}$ \\
Extraordinario de protección & & & $\mathrm{X}$ \\
\hline
\end{tabular}

Fuentes: Código de Procedimiento Penal de Chile, Código Procesal Penal de Perú y Código de Procedimiento Penal de Ecuador.

En el caso ecuatoriano, luego de la calificación de la demanda, la orden judicial de pago y la contestación por parte del deudor, el juez debe imperiosamente convocar a una audiencia orientada esencialmente a buscar un acuerdo entre las partes procesales - audiencia de conciliación - . Luego de esta diligencia, y siempre con la petición escrita del acreedor, se otorgan seis días para las diligencias probatorias. Vencido este plazo, y nuevamente mediando la voluntad expresa del acreedor, el juez está compelido a conceder cuatro días más para recibir los alegatos de las partes en litigio. Solamente cuando el procedimiento citado se cumpla el juez puede dictar sentencia. Como se observa, a diferencia del caso chileno en el que conceder el plazo probatorio es potestativo del juez o del peruano en el que en la

17 La legislación chilena establece que el plazo de prueba puede ampliarse hasta por diez días adicionales, pero solamente a petición del acreedor. 
misma audiencia se evacuan las pruebas, en Ecuador se trata de diligencias autónomas, lo que implica una mayor inversión de tiempo para alcanzar la resolución del juicio ejecutivo. Además, en el caso ecuatoriano existe un paso procesal adicional, la presentación de alegatos, que no está presente en ninguno de los otros dos países analizados. La Tabla No. 5 presenta el análisis antes descrito. ${ }^{18}$

No obstante, la idea de disminuir la corrupción judicial a través de la simplificación de los procedimientos en los juicios ejecutivos y, sobre todo, de la reducción de los recursos en los juicios penales, provocaría una aparente tensión frente a la defensa de las garantías y el debido proceso de inculpados y víctimas. El argumento que se plantea es que los recursos procesales constituyen un reaseguro para la tutela de los derechos esenciales de las personas, por lo que limitar las instancias de impugnación resultaría atentatorio contra tales derechos. Aunque dicha tensión parecería plausible, un argumento en contrario indica que la mejora en la calidad de los jueces y la disminución en la corrupción judicial precisamente a través de la economía procesal, terminará beneficiando a la ciudadanía en el plano de la seguridad jurídica y el respeto a sus derechos básicos. Los datos de la medición empírica antes descrita constan en la Tabla No. 5.

Tabla No. 5

Etapas del juicio ejecutivo en Chile, Perú y Ecuador

\begin{tabular}{cccc}
\hline Países & Chile & Perú & Ecuador \\
Etapas & & & \\
\hline Calificación de la demanda & Sí & Sí & Sí \\
Audiencia y prueba & No & Sí & No \\
Prueba & Facultativo & No & Sí \\
Alegatos & No & No & Sí \\
Sentencia & Sí & Sí & Sí \\
\hline
\end{tabular}

Fuentes: Código de Procedimiento Civil de Chile, Código Procesal Civil de Perú y Código de Procedimiento Civil de Ecuador.

Incentivos institucionales para el comportamiento ético de los jueces

Para capturar la información que permita medir esta variable se recurrió al análisis de las leyes orgánicas del Poder Judicial de Chile, Perú y Ecuador.

18 Un aspecto que es necesario destacar es que ante la ausencia de contradicción por parte del deudor, el juez dicta sentencia sin mayor trámite. Éste es un rasgo procesal que comparten los tres países analizados. 
En dichos textos legales se verificó si existían declaraciones expresas en las que se otorguen retribuciones o reconocimientos de algún tipo a los jueces con una conducta ética. Aunque se pudo constatar la existencia de disposiciones en sentido contrario, es decir, normas que señalan que el inicio de procesos judiciales o administrativos por casos de corrupción judicial trae consigo incentivos negativos para que los jueces sean ascendidos en la pirámide del Poder Judicial, no se observó ningún tipo de incentivo positivo por un comportamiento honesto. Junto a la revisión de los cuerpos legales citados se analizaron normas secundarias que podrían establecer alguna forma de "premio" al juez con conducta ética, sin hallarse evidencia alguna. Finalmente, dicho hallazgo fue corroborado por las entrevistas realizadas en los tres países analizados.

\section{Fraccionamiento del poder político}

A fin de valorar el grado de fraccionamiento del poder durante los periodos históricos seleccionados, se consideró como proxy la distribución de asientos alcanzados en la legislatura por los partidos políticos. Para ello se recurrió a la medición proporcionada por el Instituto de Estudios de Iberoamérica y Portugal de la Universidad de Salamanca, que ofrece los porcentajes de representación legislativa de cada partido político. Con dicha información se elaboró un indicador de la variable anotada que va desde " 1 ", que significa una concentración total del poder político, y concluye en " 0 ", que da cuenta del fenómeno contrario. Dicho valor se obtuvo de dividir el porcentaje de asientos alcanzados por el partido más representado para el total de la legislatura.

Cabe anotar que los datos están medidos en función de los periodos legislativos de cada país, y éstos no coinciden de forma fidedigna con los cortes temporales utilizados en este artículo. Por tanto, allí existen sesgos que no pueden ser superados. No obstante, hay una considerable cercanía entre unos periodos y otros por lo que los datos pueden ser asumidos como una buena aproximación a la realidad. Además, y al igual que en otras variables ya analizadas, las entrevistas realizadas en Santiago, Lima y Quito confirmaron las tendencias descritas en la Tabla No. 6.

En el caso chileno, la distribución del poder político durante los periodos analizados muestra una tendencia moderada y estable. No hay variaciones ostensibles aunque durante los últimos años se verifica una mayor fragmentación partidista, cuya posible explicación está en el cambio de orientación ideológica en el gobierno central a raíz de la llegada del presidente Piñera. 
Respecto de Perú, el último periodo analizado da cuenta de una drástica caída de la concentración del poder político en relación al momento histórico previo, que corresponde a la hegemonía del ex presidente Alberto Fujimori. Finalmente, en el caso ecuatoriano existe una tendencia opuesta a la antes descrita. De hecho, durante el periodo comprendido entre 2008 y la actualidad la concentración del poder político ha aumentado en relación con la observada en momentos previos. Lo dicho guarda relación con el ascenso del movimiento político Alianza País y su líder Rafael Correa. ${ }^{19}$

Tabla No. 6

\section{Fraccionamiento del poder político en Chile, Perú y Ecuador}

\begin{tabular}{cccc}
\hline País & $\begin{array}{c}\text { Periodo 1/ } \\
\text { partido más representado }\end{array}$ & $\begin{array}{c}\text { Periodo 2/ } \\
\text { partido más representado }\end{array}$ & $\begin{array}{c}\text { Periodo N 3/ } \\
\text { partido más representado }\end{array}$ \\
\hline Chile & $0.30 / \mathrm{PDC}$ & $0.32 / \mathrm{PDC}$ & $0.27 / \mathrm{UDI}$ \\
Perú & $\mathrm{n} / \mathrm{d}$ & $0.55 /$ Cambio 98 & $0.37 /$ Perú Posible \\
Ecuador & $0.28 / \mathrm{DP}$ & $0.24 / \mathrm{PSC}$ & $0.47 / \mathrm{AP}$ \\
\hline
\end{tabular}

"1" corresponde a concentración total del poder político y va hasta "0" que implica dispersión total del poder político. PDC = Partido Demócrata Cristiano. UDI = Unión Demócrata Independiente. DP $=$ Democracia Popular. PSC $=$ Partido Social Cristiano. $\mathrm{n} / \mathrm{d}=$ dato no disponible.

Fuente: Instituto de Estudios de Iberoamérica y Portugal de la Universidad de Salamanca.

Como síntesis del análisis previo, la Tabla No. 7 ofrece un modelo de comparación en el que se evidencian los factores que, por su variabilidad en los tres países analizados, explican de mejor forma la corrupción judicial en cortes intermedias e inferiores. Puesto que los cambios más importantes en el grado de corrupción judicial en Chile, Perú y Ecuador se verifican durante el último periodo estudiado, el modelo abajo descrito debe ser entendido como una comparación sincrónica. Así, la calidad de los jueces, el respeto a la carrera judicial, la cantidad de procedimientos previos a obtener una sentencia, y el grado de fragmentación del poder político constituyen algunas de las variables que explican por qué en algunos países existe mayor corrupción judicial que en otros.

En otros términos, los hallazgos empíricos ya descritos permiten afirmar que existen tres tipos de factores que afectan a la corrupción judicial.

19 Más aún, luego de las elecciones nacionales realizadas en febrero de 2013 en Ecuador, el presidente Correa no sólo resultó reelecto sino que además contará con una bancada legislativa de 100 asientos de un total de 137. 
Dentro del primer factor se encuentran aquellas variables endógenas a las cortes de justicia, como la calidad de los jueces y el respeto a la carrera judicial. En el segundo factor se hallan las variables institucionales, y específicamente aquéllas referidas al número de trámites que se deben seguir para alcanzar un fallo judicial. Finalmente, el factor restante tiene que ver con variables exógenas al funcionamiento de las cortes de justicia y, fundamentalmente, con el grado de fragmentación del poder dentro de la arena política. Aunque por la naturaleza del modelo utilizado no es posible capturar el peso específico que ejerce cada factor sobre la corrupción judicial, el análisis permite identificar de forma analítica las variables que inciden sobre el fenómeno estudiado.

Tabla No. 7

Factores que explican la corrupción judicial en Chile, Perú y Ecuador

\begin{tabular}{cccccccc}
\hline País & $\begin{array}{c}\text { Difusión } \\
\text { defallos }\end{array}$ & $\begin{array}{c}\text { Calidad de } \\
\text { los jueces }\end{array}$ & $\begin{array}{c}\text { Carrera } \\
\text { judicial }\end{array}$ & Procedimiento & $\begin{array}{c}\text { Incentivos } \\
\text { institucionales }\end{array}$ & $\begin{array}{c}\text { Fraccionamiento } \\
\text { del poder político }\end{array}$ & $\begin{array}{l}\text { Corrupción } \\
\text { judicial }\end{array}$ \\
\hline Chile & Alta & Alta & Alta & Alta & No & Alta & Baja \\
Perú & Baja & Media & Media & Media & No & Media & Media \\
Ecuador & Baja & Baja & Baja & Baja & No & Baja & Alta \\
\hline
\end{tabular}

Fuentes: Código de Procedimiento Penal de Chile, Código Procesal Penal de Perú y Código de Procedimiento Penal de Ecuador. Instituto de Estudios de Iberoamérica y Portugal de la Universidad de Salamanca. Encuestas y entrevistas a expertos.

En definitiva, mayor capacitación a los jueces, atención prioritaria a la carrera judicial como criterio de ascensos e incentivos dentro del Poder Judicial, legislaciones menos engorrosas en cuanto a pasos a seguir dentro del trámite de los juicios, y una distribución del poder político que permita mutuos controles, dan cuenta de sociedades con menores niveles de corrupción judicial. Por otro lado, los hallazgos empíricos constantes en la Tabla No. 7 dan cuenta además de que la difusión de las decisiones judiciales a través de páginas web oficiales y la generación de incentivos institucionales al comportamiento ético de los jueces no constituyen variables relevantes al momento de valorar la corrupción judicial. Si bien la primera de las variables anotadas - difusión de las decisiones judiciales a través de páginas web oficiales - presenta diferencias en los valores reportados para Chile respecto de los observados en Perú y Ecuador, el grado de varianza no es lo suficientemente amplio para inferir una relación causal específica. 


\section{Conclusiones}

Este artículo exploró los principales determinantes de la corrupción judicial en cortes intermedias e inferiores de América Latina. Para el efecto, junto a algunas de las variables más referidas por la literatura especializada se agregaron otras propuestas directamente en esta investigación. Con ello, se produjo información sistemática, comparada entre países y con perspectiva diacrónica, elementos ausentes en buena parte de los trabajos que abordan dicha temática. Así, tomando como unidades de análisis los casos de Chile, Perú y Ecuador en tres periodos históricos para cada país se constató que el grado de formación profesional de los jueces, el respeto a la carrera judicial, las características de los juicios en cuanto a simplicidad del procedimiento y la fragmentación del poder político, influyen poderosamente sobre la corrupción observada al interior de los poderes judiciales.

Como se mencionó, y a pesar de las virtudes del diseño metodológico propuesto, existen sesgos difíciles de superar. Por un lado, la incapacidad de establecer el peso específico de cada variable explicativa sobre el fenómeno estudiado; y, de otro, la dificultad para medir el peso que podría ejercer la interacción de dos o más de las variables identificadas sobre la corrupción judicial. Los problemas metodológicos anotados se deben, en buena medida, a la ausencia de información medida año con año que permita realizar un estudio estadístico más preciso. Al respecto, la generación de datos fiables y medidos con periodicidad es uno de los retos de las futuras agendas de investigación en este tema. Por otro lado, las dificultades para la medición del concepto corrupción judicial se mantienen vigentes. No obstante, haber realizado un número considerable de encuestas entre especialistas en el tema, aporta a la reducción de las distancias entre el concepto en sí mismo y su referente empírico.

Independientemente de lo dicho, el artículo deja en claro que fenómenos como el de la corrupción judicial requiere ser analizado desde distintas dimensiones. De hecho, los hallazgos empíricos que aquí se proponen dan cuenta de factores endógenos y exógenos a las cortes como influyentes en el grado de recurrencia a prácticas ilegítimas. Además, la importancia que tiene sobre la corrupción judicial el diseño institucional es otro factor que se debe resaltar. Al respecto, legislaciones simplificadas, que permitan una administración de justicia eficiente, garantizan la reducción de espacios para la corrupción. En ese plano, los costos de transacción que generan el aumento de recursos procesales no solo atentan a la celeridad procesal sino también, y esencialmente, constituyen un incentivo para la emergencia de pagos ilegítimos y otras formas de corrupción judicial. 


\section{Bibliografía}

Basabe-Serrano, Santiago, 2012, "Judges without Robes and Judicial Voting in Context of Institutional Instability: The Case of Ecuador's Constitutional Court", Journal of Latin American Studies, vol. 44, núm. 1, pp. 127-161.

Basabe-Serrano, Santiago, 2011, Jueces sin toga: Políticas judiciales y toma de decisiones en el Tribunal Constitucional del Ecuador (1997-2007), Quito, Flacso Ecuador.

Basabe-Serrano, Santiago y John Polga Hecimovich, 2013, “Legislative Coalitions and Judicial Instability: The Case of Ecuador's Constitutional Court (19992007)", Political Research Quarterly, 66, pp. 154-166.

Basabe-Serrano, Santiago, Simón Pachano y Andrés Mejía Acosta, 2010, “La democracia inconclusa: Derechos fundamentales, instituciones políticas y rendimientos gubernamentales en Ecuador (1979-2008)", Revista de Ciencia Política, vol. 30, núm. 1, pp. 65-85.

Baum, Lawrence, 1997, The Puzzle of Judicial Behavior, Michigan, The University of Michingan Press.

Buscaglia, Eduardo, 2007, "Judicial Corruption and the Broader Justice System", Global Corruption Report 2007, Cambridge, Transparency International/Cambridge University Press, pp. 67-77.

Carbonell, Miguel, 2008, El régimen constitucional de la transparencia, México, UNAM.

Carbonell, Miguel, 2007, "Judicial Corruption and Impunity in Mexico", en Global Corruption Report 2007, Cambridge, Transparency International, Cambridge University Press, pp. 225-228.

Conaghan, Catherine M., 2008, “Ecuador: Correa's Plebiscitary Presidency", Journal of Democracy, 19(2), pp. 46-60.

Conaghan, Catherine M., 2012, "Prosecuting Presidents: The Politics within Ecuador's Corruption Cases", Journal of Latin American Studies, 44(4), pp. 649678.

De la Torre, Carlos, 2010, "El gobierno de Rafael Correa: posneoliberalismo, confrontación con los movimientos sociales y democracia plebiscitaria", Revista Temas y Debates, 14(20), pp. 157-172. 
Garretón, Manuel Antonio, 2002, “La transformación de la acción colectiva en América Latina”, Revista CEPAL, núm. 76, pp. 7-24.

Gupta, Sanjeev, Hamid Davoodi y Rosa Alonso-Terme, 2002, “Does Corruption Affect Income Inequality and Poverty?", Economics of Governance, vol. 1, núm. 3, pp. 23-45.

Hammergren, Linn, 2007, “Fighting Judicial Corruption: a Comparative Perspective from Latin America", en Global Corruption Report 2007, Cambridge, Transparency International/Cambridge University Press, pp. 138-146.

Harasic, Davor, 2007, “Chile's Partial Success", en Global Corruption Report 2007, Cambridge, Transparency International/Cambridge University Press, pp. 187190.

Knaul, Gabriela, 2011, Informe de la Relatora Especial sobre la independencia de los magistrados y abogados, Adición: Misión a México. Consejo de Derechos Humanos de la ONU. Disponible en: <http://www2.ohchr.org/english/bodies/hrcouncil/ docs/17session/A-HRC-17-30-Add3_sp.pdf $>$

Melgar Peña, Carlos, 2007, “Judicial Corruption and the Military Legacy in Guatemala", en Global Corruption Report 2007, Cambridge, Transparency International/Cambridge University Press, pp. 211-214.

Montinola, G. y Jackman, R., 2002, “Sources of Corruption: A Cross Country Study”, British Journal of Political Science, núm. 32, pp. 147-170.

North, Douglas, 1993, Instituciones, cambio institucional y crecimiento económico, México, Fondo de Cultura Económica.

Pachano, Simón, 2010, "Ecuador: el nuevo sistema político en funcionamiento", Revista de Ciencia Política, vol. 30, núm. 2, pp. 297-317.

Pepys, Mary Noel, 2007, "Corruption within the Judiciary: Causes and Remedies", en Global Corruption Report 2007, Cambridge, Transparency International/Cambridge University Press, pp. 3-11.

Przeworski, Adam y Henry Teune, 1970, The logic of Comparative Social Inquiry, WileyInterscience, New York, John Wiley \& Sons.

Robertson, C., y Watson, A., 2004, “Corruption and Change: The Impact of Foreign Direct Investment”, Strategic Managment Journal, vol. 25, núm. 4, pp. 385-396. 
Rose-Ackerman, Susan, 2007, "Judicial Independence and Corruption”, en Global Corruption Report 2007, Cambridge, Transparency International/ Cambridge University Press, pp.15-24.

Salazar, Roxana y José Pablo Ramos, 2007, “Increased Transparency Helps Curb Corruption in Costa Rica", en Global Corruption Report 2007, Cambridge, Transparency International/Cambridge University Press, pp. 190-194.

Sánchez Urribarrí, Raúl, 2008, “Characteristics of the Judiciary vs. Corruption Perception", Sistemas Judiciales, 13, pp. 88-99.

Selligson, Mitchell, 2002, “The Impact of Corruption on Regime Legitimacy: A Comparative Study of Four Latin American Countries", Journal of Politics, vol. 64, núm. 2, pp. 408-433.

Tsebelis, George, 2002, Veto Players: how Political Institutions Work, Princeton, Princeton University Press.

Voigt, Stefan, 2007, “When are Judges Likely to be Corrupt?", en Global Corruption Report 2007, Cambridge, Transparency International/Cambridge University Press, pp. 296-301.

Yang, Vincent y Linda Ehrichs, 2007, “The Professionalism of Judges: Education, Salaries and Career Structure in Asia", en Global Corruption Report 2007, Cambridge, Transparency International/Cambridge University Press, pp. 48-56.

Recibido el 31 de mayo de 2012

Aceptado el 29 de mayo de 2013 